\title{
Therapeutic Effect of Fimasartan in a Rat Model of Myocardial Infarction Evaluated by Cardiac Positron Emission Tomography with $\left[{ }^{18}\right.$ F]FPTP
}

\author{
Hyukjin Park ${ }^{1, \dagger}$, Hyeon Sik Kim ${ }^{2, \dagger}$, Young Joon Hong ${ }^{1, *}$, Jung-Joon Min ${ }^{3, *}$, Han Byul Kim ${ }^{1}$, \\ Min Chul Kim ${ }^{1}$, Doo Sun Sim ${ }^{1}$, Ju Han Kim ${ }^{1}$, Dong-Yeon Kim ${ }^{3}$, Jae Sung Lee ${ }^{4}$, Youngkeun Ahn ${ }^{1}$, \\ and Myung Ho Jeong ${ }^{1}$
}

${ }^{1}$ Division of Cardiology, Chonnam National University Hospital, Gwangju, ${ }^{2}$ Institute for Biomedical Science, Chonnam National University Hwasun Hospital, ${ }^{3}$ Department of Nuclear Medicine, Chonnam National University Hwasun Hospital, Hwasun, ${ }^{4}$ Department of Nuclear Medicine, Seoul National University Hospital, Seoul, Korea

We evaluated the efficacy of fimasartan on perfusion defects and infarction size in an animal model of myocardial infarction (MI), with echocardiography and positron emission tomography (PET) using a ${ }^{18} \mathrm{~F}$-labeled phosphonium cation $\left(5-\left[{ }^{18} \mathrm{~F}\right]\right.$-fluoropentyltriphenylphosphonium salt, $\left.\left[{ }^{18} \mathrm{~F}\right] \mathrm{FPTP}\right)$ as a mitochondrial voltage sensor for myocardial imaging. We induced MI in 33 rats by ligation of the left coronary artery, and checked their cardiac PET image using $\left[{ }^{18} \mathrm{~F}\right]$ FPTP for evaluation of myocardial perfusion. Rats were grouped into 3 groups according to their administered drugs: no drug $(\mathrm{n}=11)$, fimasartan $3 \mathrm{mg} / \mathrm{kg}(\mathrm{n}=10)$, and fimasartan $10 \mathrm{mg} / \mathrm{kg}(\mathrm{n}=12)$. Each designated drug was administered for 4 weeks, and follow-up PET and histologic examinations were done. In the PET analysis, a perfusion defect size was markedly improved in fimasartan $10 \mathrm{mg} / \mathrm{kg}$ group $(35.9 \pm 7.0 \%$ to $28.4 \pm 6.9 \%$, p $<0.001)$, whereas treatment with fimasartan $3 \mathrm{mg} / \mathrm{kg}$ induced only an insignificant reduction of perfusion defect size $(35.9 \pm 7.9 \%$ to $33.9 \pm 7.3 \%, \mathrm{p}=0.095)$. Using $2,3,5$-triphenyltetrazolium chloride staining, infarction size was the largest in the control group (36.5 $\pm 8.3 \%)$, and was insignificantly lower in the fimasartan $3 \mathrm{mg} / \mathrm{kg}$ group $(31.5 \pm 6.5 \%$, p for the difference between the control group $=0.146$ ) and was significantly lower in the fimasartan $10 \mathrm{mg} / \mathrm{kg}$ group $(26.3 \pm 7.6 \%, \mathrm{p}$ for the difference between the control group $=0.011)$. PET imaging using $\mathrm{a}{ }^{18} \mathrm{~F}$-labeled mitochondrial voltage sensor, $\left[{ }^{18} \mathrm{~F}\right] \mathrm{FPTP}$, is useful in evaluation and monitoring of myocardial perfusion states, and treatment with fimasartan decreases the infarction size in animal MI model.

Key Words: Myocardial Infarction; Positron Emission Tomography; Angiotensin Receptor Antagonists

This is an Open Access article distributed under the terms of the Creative Commons Attribution Non-Commercial License (http://creativecommons.org/licenses/by-nc/4.0) which permits unrestricted non-commercial use,

distribution, and reproduction in any medium, provided the original work is properly cited.

\section{Article History:}

Received January 23, 2019

Revised March 13, 2019

Accepted April 2, 2019

\section{Corresponding Author:}

Young Joon Hong

Division of Cardiology, Cardiovascular

Convergence Research Center

Nominated by Korea Ministry of Health

and Welfare, Chonnam National

University Hospital, 42 Jaebong-ro,

Dong-gu, Gwangju 61469, Korea

Tel: +82-62-220-6978

Fax: +82-62-223-3105

E-mail: hyj200@hanmail.net

\section{Jung-Joon Min}

Department of Nuclear Medicine,

Chonnam National University Hwasun Hospital, 322 Seoyang-ro,

Hwasun-eup, Hwasun 58128, Korea

Tel: +82-61-379-2876

E-mail: jjmin@jnu.ac.kr

${ }^{\dagger}$ These authors contributed equally to this work.

\section{INTRODUCTION}

Not all affected myocardial tissue is irreversibly damaged during acute myocardial infarction (MI). Dysfunctional myocardial tissue occurring during acute MI consists of necrotic, hibernating, or stunned myocardial cells, and the viable (hibernating or stunned) tissue can be salvaged by revascularization. ${ }^{1}$ On the other hand, totally infarcted myocardial tissue, with prolonged and fixed perfusion de- fect cannot be easily restored and therefore, is not always indicated for revascularization. Therefore, the extent of viable myocardium is important for recovery of cardiac function and prognosis.

To detect myocardial activity and evaluate the viability of myocardium, various imaging modalities, such as echocardiography, cardiac magnetic resonance imaging (MRI), single photon emission computed tomography (SPECT), and positron emission tomography (PET) have been used, 
but each modality has its limitations: echocardiography is highly dependent on the echocardiographic windows and cutting plane, MRI cannot easily distinguish between epicardial fat layers and the myocardium and have limitations in evaluating myocardial perfusion, SPECT has low spatial resolution, and an unintended spread of the SPECT tracers to adjacent organs may compromise accurate diagnosis. ${ }^{2}$

PET is known to provide more accurate and highly qualified images due to its higher spatial resolution, and may enable quantitative measurements of myocardial tracer uptake. ${ }^{3}$ In particular, our group previously proposed the value of ${ }^{18} \mathrm{~F}$-labeled phosphonium cations as myocardial imaging agents that accumulate in cardiomyocytes as a result of the higher mitochondrial membrane potential (MMP), and our group reported that $\left[{ }^{18} \mathrm{~F}\right] \mathrm{FPTP}$ provided excellent image quality when compared with $\left[{ }^{13} \mathrm{~N}\right] \mathrm{NH}_{3}$ in normal and MI rats. ${ }^{2,4,5}{ }^{18} \mathrm{~F}$-labeled phosphonium cations accurately evaluated the size of MI early after tracer injection, and yielded excellent image quality in a rat model of coronary occlusion. ${ }^{5}$

As the renin-angiotensin-aldosterone system (RAAS) plays an important role in tissue fibrosis, cardiac remodeling, fluid and sodium accumulation, and inflammation, the blockade of RAAS has been a standard therapy in both heart failure and MI. ${ }^{6}$ Fimasartan, a selective type 1 angiotensin II (AT1) receptor blocker, has shown good tolerability and blood pressure-lowering effect for hypertension $^{7,8}$ and has shown efficacy in rat doxorubicin-induced cardiotoxicity models. ${ }^{9}$ However, to date, its efficacy in MI has not been well demonstrated, only conflicting results exist, ${ }^{10,11}$ and no study has examined its efficacy in cardiac PET using ${ }^{18} \mathrm{~F}$-labeled phosphonium cations yet.

We aimed both to verify anti-adverse remodeling effect of fimasartan, and also to evaluate the efficacy of cardiac PET using a ${ }^{18} \mathrm{~F}$-labeled phosphonium cation, $\left(5-\left[{ }^{18} \mathrm{~F}\right]\right.$ fluoropentyl)triphenylphosphonium salt ( $\left.\left[{ }^{18} \mathrm{~F}\right] \mathrm{FPTP}\right)$, in measuring area of perfusion defect in rat $\mathrm{MI}$ model after the MI event and after medical treatment.

\section{MATERIALS AND METHODS}

\section{MI induction and imaging studies}

All procedures were performed in accordance with 1964 Helsinki Declaration and its later amendments or comparable ethical standards. Induction of MI and breeding were conducted at the animal laboratory of Chonnam
National University Hospital, Gwangju, South Korea. The study protocol was approved by the Institutional Review Board at Chonnam National University Hospital. The study protocol is illustrated in Fig. 1. Eight-week old male Sprague-Dawley rats $(\mathrm{n}=33$ ) with body weights between 240-290 g were used for the experiment. Anesthesia was performed with ketamine $100 \mathrm{mg} / \mathrm{kg}$ and xylazine 10 $\mathrm{mg} / \mathrm{kg}$. After endotracheal intubation, thoracotomy and pericardiotomy, left coronary artery (LCA) was ligated with 5-0 silk. Rats were observed for 7 days after MI induction, and then echocardiography and PET were checked.

\section{Echocardiography}

A conventional echocardiography system with a linear array transducer (Vivid S5, GE Healthcare, Wauwatosa, WI, USA) was used for echocardiography. Echocardiography was checked while in the supine position after shaving off the chest hair of the subjects. We acquired short-axis images of the left ventricle (LV) by transthoracic echocardiography, and a M-mode LV study was done to measure the parameters of the $L V$, including $L V$ ejection fraction (LVEF), LV end-diastolic diameter (LVEDD), and LV end-systolic diameter (LVESD).

\section{PET image acquisition}

After conducting the MI induction and checking the baseline echocardiography, cardiac PET images using $\left[{ }^{18} \mathrm{~F}\right]$ FPTP were acquired by microPET scanner (Inveon. Siemens Medical Solutions, Malvern, PA, USA). $\left[{ }^{18}\right.$ F]FPTP was prepared following a previously described method. ${ }^{4}$ The total reaction time of $\left[{ }^{18} \mathrm{~F}\right] \mathrm{FPTP}$ was less than 60 minutes, the overall decay-corrected radiochemical yield was about $15-20 \%$, and the radiochemical purity was $>98 \%$. $10 \mathrm{mi}-$ nute, static PET images were acquired at 10 minutes and 30 minutes after intravenous injection of $\left[{ }^{18} \mathrm{~F}\right]$ FPTP. A dedicated microPET was used for in vivo imaging of rats that were anesthetized with isoflurane, placed in a cradle, and equipped with masks for anesthetic gas supply. Acquired images were reconstructed using a 3D-ordered subset expectation maximization (3D-OSEM) algorithm with four iterations. Reconstructed pixel sizes were $0.78 \mathrm{~mm}$ in the transverse and axial directions. The dimensions of the reconstructed images were $128 \times 128$ pixels in each of the 159 transverse slices. Data were normalized and corrected for randomness, dead time, and decay. Myocardial perfusion defect was clearly defined with good heart-to-liver and heart-to-lung contrast.

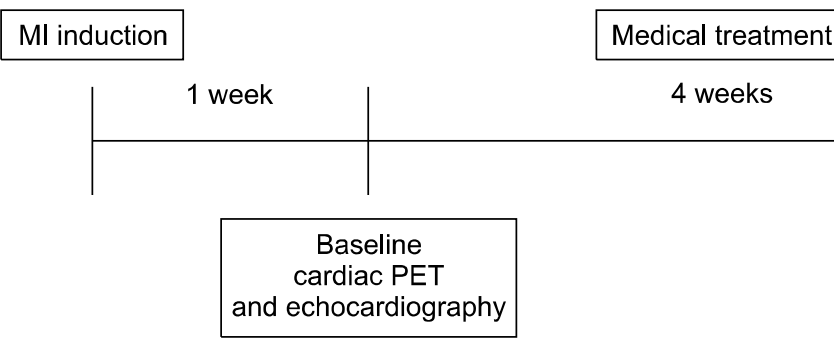

Sacrificed

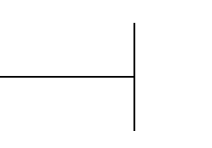

Follow-up cardiac PET and echocardiography
Fig. 1. Study protocol. MI: myocardial infarction, PET: positron emission tomography. 


\section{Medical treatment and follow-up studies}

After MI induction and checking baseline echocardiography and PET, medical treatment was provided to rats. Rats were grouped into 3 groups according to their prescribed drugs: no drug ( $\mathrm{n}=11)$, fimasartan $3 \mathrm{mg} / \mathrm{kg}(\mathrm{n}=10)$, fimasartan $10 \mathrm{mg} / \mathrm{kg}(\mathrm{n}=12)$. Drug dose was set by referring previous reports. ${ }^{9,11}$ Each designated drug was dissolved in $10 \mathrm{~mL}$ of water and was administered via transoral route using a zonde. Drug administration was done every day at 11:00 AM, for 4 weeks. After the treatment period, followup echocardiography and cardiac PET were checked, in the same way as the baseline studies. Then the rats were sacrificed by injecting $1 \mathrm{~mL}(40 \mathrm{mEq} / 20 \mathrm{~mL})$ of potassium chloride solution into the abdominal aorta, and their hearts were extracted using 2, 3, 5-triphenyltetrazolium chloride (TTC) staining. The extracted heart tissue was divided into three $2 \mathrm{~mm}$-thick slices vertically from the cardiac apex to the ligation site. The sliced heart tissue was transferred into a $50 \mathrm{~mL}$ tube with 5-10 mL warmed 1\% TTC solution and kept in the water bath with gentle shaking for around 5-10 min. It was reviewed by a pathologist and the infarction size was measured on digital photographs, by outlining the LV area and TTC negative infarction area, and the size of infarction was reported as a percentage of total LV area. Results of the TTC stain were compared with the size of perfusion defect in polar maps of cardiac PET (Fig. 2).

\section{Image analysis and statistical analysis}

PMOD version 3.310 (PMOD Technologies Ltd., Zurich, Switzerland) was used for image analysis which is SUV calculation, image display and cardiac polar map analysis (60\% threshold). We used Statistical Package for the Social Sciences (SPSS) 22.0 for Microsoft Windows (SPSS, Inc., Chicago, IL, USA) for all statistical analyses. All numerical variables were presented as mean value \pm standard deviation (SD) and were compared by a paired t-test. Furthermore, a Pearson's correlation analysis was used to figure out the correlation between perfusion defect size in PET and infarction size in TTC stain. A p value $<0.05$ was considered statistically significant.

\section{RESULTS}

\section{Baseline and follow-up echocardiographic results}

Baseline (1 week after MI induction) and follow-up (after treatment for 4 weeks) echocardiographic results are shown in Table 1. At the baseline, no significant differences in echocardiographic parameters between groups were observed. The control group experienced significant LV dilatation (LVEDD changes: $0.87 \pm 0.09 \mathrm{~cm}$ to $1.01 \pm 0.09 \mathrm{~cm}$, $\mathrm{p}=0.002)$ and significant LVEF impairment $(44.82 \pm 5.25 \%$ to $37.29 \pm 8.02 \%, \mathrm{p}=0.001$ ). On the other hand, treatment with fimasartan resulted in better outcomes: insignificant LV dilation (LVEDD changes: $0.91 \pm 0.10 \mathrm{~cm}$ to $0.96 \pm 0.14$
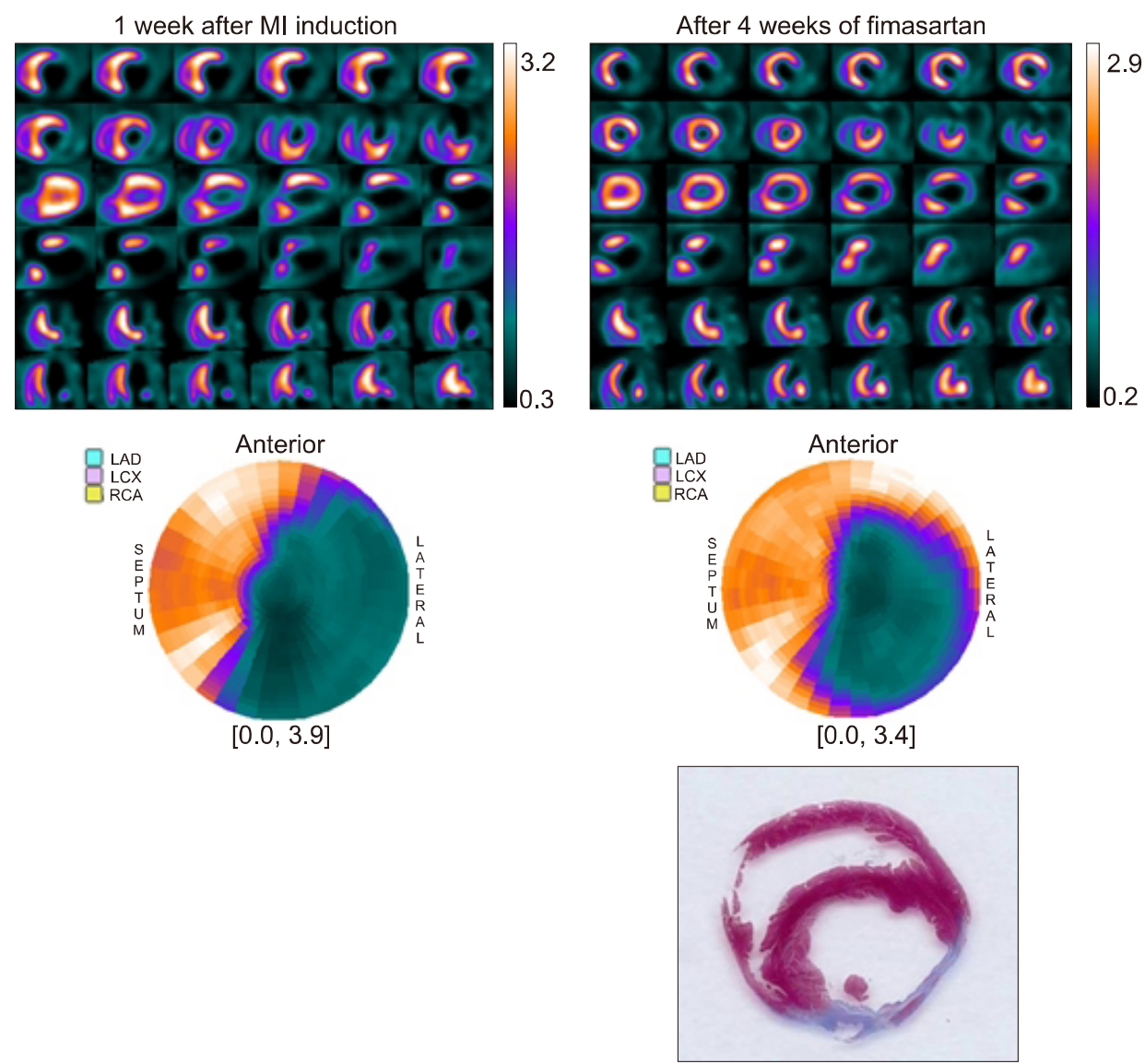

Fig. 2. Representative PET images in polar map 1 week after myocardial infarction induction (left) and after 4 weeks of fimasartan treatment (right). Short axis section of the extracted heart is illustrated in right lower side. 
TABLE 1. Baseline and follow-up echocardiographic Findings

\begin{tabular}{lcccc}
\hline Baseline (1 week after MI induction) & Control $(\mathrm{n}=11)$ & Fimasartan $3 \mathrm{mg} / \mathrm{kg}(\mathrm{n}=10)$ & Fimasartan $10 \mathrm{mg} / \mathrm{kg}(\mathrm{n}=12)$ & $\mathrm{p}$ value \\
\hline LVEDD (cm) & $0.87 \pm 0.09$ & $0.91 \pm 0.10$ & $0.90 \pm 0.13$ & 0.134 \\
LVESD (cm) & $0.70 \pm 0.09$ & $0.75 \pm 0.10$ & $0.73 \pm 0.11$ & 0.107 \\
LVEF (\%) & $44.82 \pm 5.25$ & $42.35 \pm 4.91$ & $43.75 \pm 2.93$ & 0.240 \\
\hline Follow-up (after treatment for 4 weeks) & Control (n=11) & Fimasartan $3 \mathrm{mg} / \mathrm{kg}(\mathrm{n}=10)$ & Fimasartan $10 \mathrm{mg} / \mathrm{kg}(\mathrm{n}=12)$ & $\mathrm{p}$ value \\
\hline LVEDD (cm) & $1.01 \pm 0.09$ & $0.96 \pm 0.14$ & $0.95 \pm 0.09$ & 0.101 \\
LVESD (cm) & $0.85 \pm 0.10$ & $0.77 \pm 0.13$ & $0.75 \pm 0.08$ & 0.067 \\
LVEF (\%) & $37.29 \pm 8.02$ & $44.02 \pm 6.33$ & $47.58 \pm 3.80$ & $<0.001$ \\
\hline
\end{tabular}

MI: myocardial infarction, LVEDD: left ventricular end-diastolic diameter, LVESD: left ventricular end-systolic diameter, LVEF: left ventricular ejection fraction.
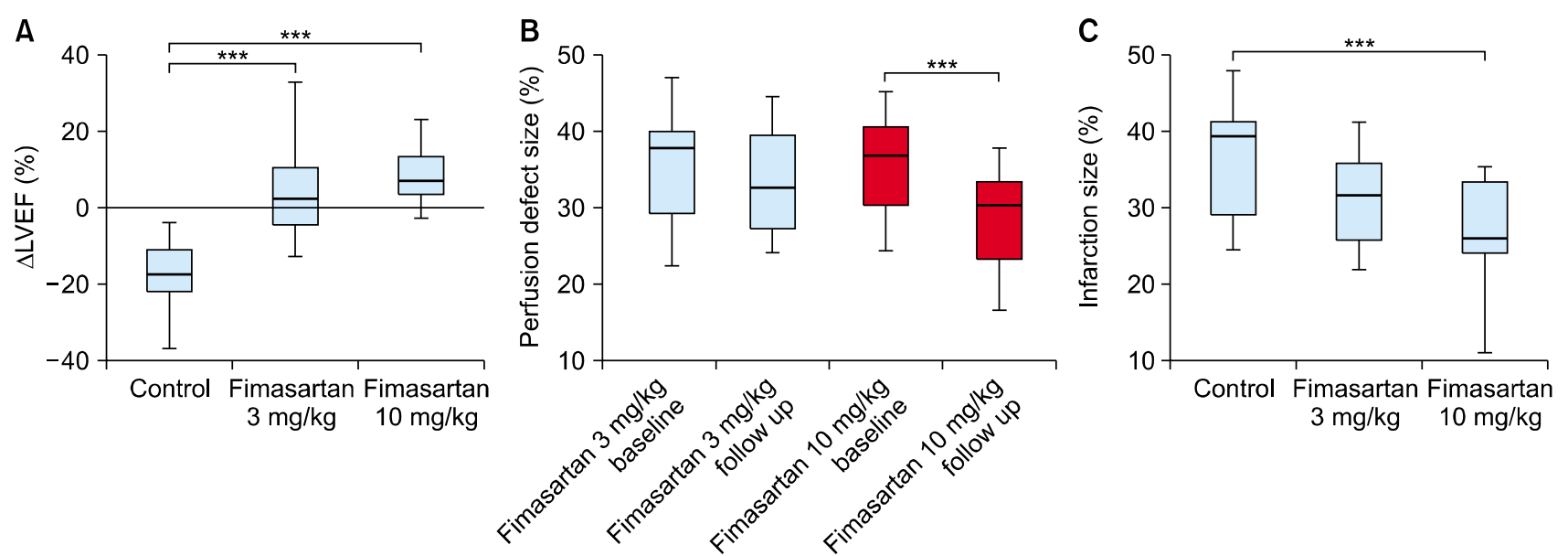

FIG. 3. (A) $\triangle$ LVEF [(LVEF in follow-up echocardiography - LVEF in baseline echocardiography)/LVEF in baseline echocardiography] in 3 groups. (B) Mean perfusion defect size in baseline and follow-up cardiac PET. (C) Mean infarction size in TTC stain. Asterisks between groups mean statistically significant difference between them $(\mathrm{p}<0.05)$.

$\mathrm{cm}, \mathrm{p}=0.649)$ and insignificant LVEF improvement $(42.35 \pm$ $4.91 \%$ to $44.02 \pm 6.33 \%$, $\mathrm{p}=0.07$ ) were observed in fimasar$\tan 3 \mathrm{mg} / \mathrm{kg}$ group, and treatment with fimasartan 10 $\mathrm{mg} / \mathrm{kg}$ showed marginal dilation of $\mathrm{LV}(0.90 \pm 0.13 \mathrm{~cm}$ to $0.95 \pm 0.09 \mathrm{~cm}, \mathrm{p}=0.625$ ) and no significant change in $\mathrm{LVEF}$ $(43.75 \pm 2.93 \%$ to $47.58 \pm 3.80 \%, \mathrm{p}=0.089)$.

\section{Changes of LVEF from baseline to follow-up}

$\triangle \mathrm{LVEF}$, which is defined as [(LVEF in follow-up echocardiography - LVEF in baseline echocardiography) / LVEF in baseline echocardiography], is illustrated in Fig. 3A. It was significantly different between the control and the fimasartan $3 \mathrm{mg} / \mathrm{kg}$ group $(-17.4 \pm 10.1 \%$ vs. $4.3 \pm 13.6 \%$, $\mathrm{p}=0.001$ ), and so it was between the control and the fimasartan $10 \mathrm{mg} / \mathrm{kg}$ group $(-17.4 \pm 10.1 \%$ vs. $8.1 \pm 7.3 \%, \mathrm{p}<0.001)$.

\section{Baseline and follow-up cardiac PET results}

Baseline (1 week after MI induction) and follow-up (after treatment for 4 weeks) cardiac PET results are compared in polar maps (representative images in Fig. 2) and are illustrated in Fig. 3B. Also, the values are presented in Table 2. At the baseline, no significant differences were observed between the perfusion defect size between the fimasartan
TABLE 2. Baseline and follow-up PET Findings after MI induction and after treatment

\begin{tabular}{lccc}
\hline Perfusion defect (\%) & $\begin{array}{c}\text { Fimasartan } \\
3 \mathrm{mg} / \mathrm{kg}(\mathrm{n}=10)\end{array}$ & $\begin{array}{c}\text { Fimasartan } \\
10 \mathrm{mg} / \mathrm{kg}(\mathrm{n}=12)\end{array}$ & $\mathrm{p} \mathrm{value}$ \\
\hline $\begin{array}{c}\text { Baseline (1 week after } \\
\text { MI induction) }\end{array}$ & $35.9 \pm 7.9$ & $35.9 \pm 7.0$ & 0.985 \\
$\begin{array}{c}\text { Follow-up (after treat- } \\
\text { ment for 4 weeks) }\end{array}$ & $33.9 \pm 7.3$ & $28.4 \pm 6.9$ & 0.108 \\
\hline
\end{tabular}

PET: positron emission tomography, MI: myocardial infarction.

$3 \mathrm{mg} / \mathrm{kg}$ group and the fimasartan $10 \mathrm{mg} / \mathrm{kg}$ group $(35.9 \pm$ $7.9 \%$ vs. $35.9 \pm 7.0 \%, \mathrm{p}=0.985)$. The perfusion defect size was markedly decreased in the fimasartan $10 \mathrm{mg} / \mathrm{kg}$ group $(35.9 \pm 7.0 \%$ to $28.4 \pm 6.9 \%, \mathrm{p}<0.001)$, whereas treatment with fimasartan $3 \mathrm{mg} / \mathrm{kg}$ induced only insignificant reduction of perfusion defect size $(35.9 \pm 7.9 \%$ to $33.9 \pm 7.3 \%$, $\mathrm{p}=0.095)$ from baseline to follow-up.

\section{TTC staining results}

In TTC staining, the infarction size was the largest in the control group $(36.5 \pm 8.3 \%)$, and it was insignificantly lower 


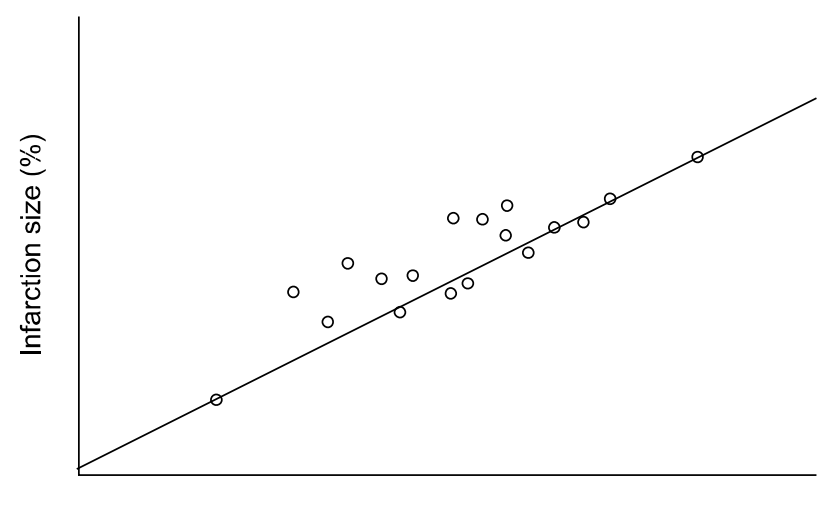

Perfusion defect size (\%)

FIG. 4. Relation between infarction size in TTC stain (\%) and perfusion defect size in PET (\%). Partial correlation coefficient $(r)=0.89$.

in fimasartan $3 \mathrm{mg} / \mathrm{kg}$ group $(31.5 \pm 6.5 \%$, p for difference between the control group=0.146) and was significantly lower in the fimasartan $10 \mathrm{mg} / \mathrm{kg}$ group $(26.3 \pm 7.6 \%, \mathrm{p}$ for the difference between the control group=0.011) (Fig. 3C). The partial correlation coefficient $(r)$ for perfusion defect size in follow-up cardiac PET and infarction sized in TTC staining was 0.89 , showing strong correlation between them (Fig. 4).

\section{DISCUSSION}

This study demonstrated usefulness of cardiac PET using $\left[{ }^{18} \mathrm{~F}\right] \mathrm{FPTP}$ as a myocardial imaging agent to precisely predict perfusion defect size and to monitor changes of myocardial perfusion state after treatment. And it also demonstrated that treatment with fimasartan in rat MI model can both preserve LV function and reduce infarction size in echocardiographic, PET, and histologic findings.

Despite the advantages of PET in image quality, its use has been limited by several factors, such as short half-life of previously developed PET tracers, such as ${ }^{82} \mathrm{Rb}$ (1.27 minutes), $\left[{ }^{15} \mathrm{O}\right] \mathrm{H}_{2} \mathrm{O}$ (2.03 minutes), and $\left[{ }^{13} \mathrm{~N}\right] \mathrm{NH}_{3}(9.97 \mathrm{mi}-$ nutes), and temporal / spatial limitations since a cyclotron or a generator is required nearby. ${ }^{12,13}$ On the contrary, ${ }^{18} \mathrm{~F}$-labeled tracers have longer half-lives (109.8 minutes), allowing convenient clinical uses. Furthermore ${ }^{18} \mathrm{~F}$-labeled phosphonium cations can be absorbed more selectively by cardiomyocytes than by other cells, due to their higher MMP, resulting in good myocardium-to-background (such as lung or liver) ratios and consequent sharp definition and good image quality. ${ }^{4}$ Also, since MMP is lost in the early phase of cell death by myocardial ischemia or infarction, ${ }^{14}$ use of ${ }^{18} \mathrm{~F}$-labeled tracers can be useful for detecting any cell death in MI. After introduction of the first ${ }^{18} \mathrm{~F}$-labeled phosphonium cation, ${ }^{18} \mathrm{~F}$-fluorobenzyltriphenylphosphonium, several ${ }^{18} \mathrm{~F}$-labeled phosphonium cations were investigated, including $\left[{ }^{18} \mathrm{~F}\right]$ FPTP. Its lipophilicity and delocalized positive charge allow the cation to cross lipid bilayer by passive diffusion, and to accumulate in cells in a MMPdependent manner. Its utility and stability as a novel volt- age sensor, and superiority to $\left[{ }^{13} \mathrm{~N}\right] \mathrm{NH}_{3}$ for cardiac PET have been previously described. ${ }^{2,4,5}$

The present study suggests the utility of $\left[{ }^{18} \mathrm{~F}\right]$ FPTP in an animal MI model, showing improvement of perfusion defect size in serial cardiac PET images after treatment with fimasartan. Just as in the previous report, perfusion defect size in follow-up cardiac PET and size of infarction in TTC staining showed good correlation. ${ }^{2}$ Although these findings cannot be simply correlated, the fimasartan treatment groups had better cardiac systolic function and minimized LV dilatation in serial echocardiography, and experienced more reduction of perfusion defect size in serial cardiac PET (only in fimasartan $10 \mathrm{mg} / \mathrm{kg}$ group), all of which are indicative of the favorable effects of fimasartan, that can be monitored by serial cardiac PET images.

Use of an angiotensin converting enzyme inhibitor or an angiotensin receptor blocker is encouraged in both heart failure and MI patients according to current guidelines because of their beneficial effects against several of the harmful effects of RAAS, including tissue fibrosis, cell apoptosis, cardiac remodeling, fluid and sodium accumulation, and inflammation. ${ }^{6,15-17}$ Our group recently reported the beneficial effects of RAAS inhibition on cardiac function, structure, and clinical event-free survival in a large number of latecomer patients with MI in the Korea Acute Myocardial Infarction Registry, whose myocardial salvage and recovery by percutaneous coronary intervention are not expected to be highly effective: RAAS inhibition is even more important in this subset of the population. ${ }^{18}$ Fimasartan has been proven to have potent antihypertensive effects and already has been used as an AT1 receptor blocker for several years, ${ }^{19,20}$ but its cardioprotective effect for MI or other types of heart failure has not yet clearly been defined. Fimasartan preconditioning showed the potential to reduce mitochondrial damage in rat ischemia/reperfusion model, but it did not result in a reduction of perfusion defects in SPECT nor a reduction of infarction size in another porcine model study. ${ }^{10,11}$ Of all potential factors that could have caused these differences between these studies including the present study, we hypothesize that there is significant dose-dependent relation in the myocardial protection effect of fimasartan. For the treatment of heart failure, including ischemic heart failure, up-titration to the maximum tolerated evidence-based doses of RAAS inhibitors and beta-blockers is recommended. ${ }^{6}$ In the porcine model study cited above, the authors used relatively low doses of RAAS inhibitors (perindopril $2 \mathrm{mg}$, valsartan 40 $\mathrm{mg}$, fimasartan $30 \mathrm{mg}$ daily in $20-25 \mathrm{~kg}$ pigs), which did not result in significant therapeutic effects. ${ }^{10}$ However, preconditioning with relatively higher doses of fimasartan (3 $\mathrm{mg} / \mathrm{kg} /$ day) before the induction of myocardial ischemia/reperfusion or hypoxia/reperfusion seemed to improve cardiac performance, reduce apoptotic cell death and the collapse of membrane potential, as well as suppress mitochondrial calcium ion overload during reoxygenation. ${ }^{11}$ Those findings correlate well with previous reports, which suggested a correlation between hemodynamic recovery and 
infarction size reduction by angiotensin receptor blockers and the prevention of collapse of MMP. ${ }^{21}$ Our results reproduced those previously reported beneficial effects of fimasartan treatment by imaging studies in a dose-dependent manner, suggesting fimasartan as an effective treatment option after an occurrence of MI, especially in higher doses.

The present study has some limitations. First, although our results suggest that the current preclinical findings may be applied in clinical situations because of the stable uptake and excellent pharmacokinetics of $\left[{ }^{18} \mathrm{~F}\right] \mathrm{FPTP}$, the present study was only an experimental study in rodent models. Second, the present study was limited to acute MI with permanent LCA ligation. This model was well suited to determine myocardial defects but is not identical to the clinical situation in which hemodynamically relevant stenosis is unmasked by a stress-induced increase of myocardial blood flow. Further studies are needed to validate $\left[{ }^{18} \mathrm{~F}\right]$ FPTP PET for the detection of small myocardial ischemia and scars of infarctions. Third, each designated drug was administered for 4 weeks and follow-up studies were checked. Therefore, we did not have data for a longer follow-up period. Fourth, as we did not perform any assessment of myocardial mechanics, our results are confined to show general myocardial function and perfusion status, while mechanical function of myocardial cells was not assessed.

In conclusion, $\left[{ }^{18} \mathrm{~F}\right] \mathrm{FPTP}$, a new ${ }^{18} \mathrm{~F}$-labeled phosphonium cation is a useful cardiac PET imaging agent in MI settings as it provides precise information about perfusion defects at the baseline period and after medical treatment, and because of its clinical convenience due to its long half-life. Treatment with a new angiotensin II receptor blocker, fimasartan, preserves LV function and reduces infarction size in the rat MI model, suggesting its utility as an effective treatment option in patients with MI. Further investigations regarding this new imaging technique and this new drug have to be conducted, and their utility and efficacy remain to be proven, in larger animal models.

\section{ACKNOWLEDGEMENTS}

This study was supported by a a grant of the Korean Health Technology R\&D Project, Ministry of Health and Welfare, Republic of Korea (HI17C2150), a grant of the Korean Health Technology R\&D Project, Ministry of Health and Welfare, Republic of Korea (HI14C2069), a grant of the Korean Healt Technology R\&D Project, Ministry of Health and Welfare, Republic of Korea (HI13 C1527), a grant of the Bio \&Medical Technology Development Program of the National Research Foundation (NRF) funded by the Korean government (MEST) (2012M3A9C 6049744) and a grant of the Korean Health Technology R\&D Project, Ministry of Health and Welfare, Republic of Korea (HI13C0163).

\section{CONFLICT OF INTEREST STATEMENT}

None declared.

\section{REFERENCES}

1. Brundage BH, Massie BM, Botvinick EH. Improved regional ventricular function after successful surgical revascularization. J Am Coll Cardiol 1984;3:902-8.

2. Kim DY, Kim HS, Jang HY, Kim JH, Bom HS, Min JJ. Comparison of the cardiac microPET images obtained using [(18)F]FPTP and [(13)N]NH3 in rat myocardial infarction models. ACS Med Chem Lett 2014;5:1124-8.

3. Yoshinaga K, Chow BJ, Williams K, Chen L, deKemp RA, Garrard $\mathrm{L}$, et al. What is the prognostic value of myocardial perfusion imaging using rubidium-82 positron emission tomography? J Am Coll Cardiol 2006;48:1029-39.

4. Kim DY, Kim HS, Le UN, Jiang SN, Kim HJ, Lee KC, et al. Evaluation of a mitochondrial voltage sensor, (18F-fluoropentyl) triphenylphosphonium cation, in a rat myocardial infarction model. J Nucl Med 2012;53:1779-85.

5. Kim DY, Kim HS, Reder S, Zheng JH, Herz M, Higuchi T, et al. Comparison of $18 \mathrm{~F}$-labeled fluoroalkylphosphonium cations with 13N-NH3 for PET myocardial perfusion imaging. J Nucl Med 2015;56:1581-6.

6. Ponikowski P, Voors AA, Anker SD, Bueno H, Cleland JGF, Coats AJS, et al.; ESC Scientific Document Group. 2016 ESC guidelines for the diagnosis and treatment of acute and chronic heart failure: the Task Force for the diagnosis and treatment of acute and chronic heart failure of the European Society of Cardiology (ESC) developed with the special contribution of the Heart Failure Association (HFA) of the ESC. Eur Heart J 2016;37:2129-200.

7. Kim JH, Lee JH, Paik SH, Kim JH, Chi YH. Fimasartan, a novel angiotensin II receptor antagonist. Arch Pharm Res 2012;35: 1123-6.

8. Lee JH, Yang DH, Hwang JY, Hur SH, Cha TJ, Kim KS, et al. A randomized, double-blind, candesartan-controlled, parallel group comparison clinical trial to evaluate the antihypertensive efficacy and safety of fimasartan in patients with mild to moderate essential hypertension. Clin Ther 2016;38:1485-97.

9. Chang SA, Lim BK, Lee YJ, Hong MK, Choi JO, Jeon ES. A novel angiotensin type I receptor antagonist, fimasartan, prevents doxorubicin-induced cardiotoxicity in rats. J Korean Med Sci 2015; 30:559-68.

10. Sim DS, Jeong MH, Song HC, Kim J, Chong A, Bom HS, et al. Cardioprotective effect of fimasartan, a new angiotensin receptor blocker, in a porcine model of acute myocardial infarction. $J$ Korean Med Sci 2015;30:34-43.

11. Han J, Park SJ, Thu VT, Lee SR, Long le T, Kim HK, et al. Effects of the novel angiotensin II receptor type I antagonist, fimasartan on myocardial ischemia/reperfusion injury. Int J Cardiol 2013; 168:2851-9.

12. Siegrist PT, Husmann L, Knabenhans M, Gaemperli O, Valenta I, Hoefflinghaus T, et al. (13)N-ammonia myocardial perfusion imaging with a PET/CT scanner: impact on clinical decision making and cost-effectiveness. Eur J Nucl Med Mol Imaging 2008; 35:889-95. 
13. Sampson UK, Dorbala S, Limaye A, Kwong R, Di Carli MF. Diagnostic accuracy of rubidium-82 myocardial perfusion imaging with hybrid positron emission tomography/computed tomography in the detection of coronary artery disease. J Am Coll Cardiol 2007;49:1052-8.

14. Chen LB. Mitochondrial membrane potential in living cells. Annu Rev Cell Biol 1988;4:155-81.

15. Toh N, Ishii K, Kihara H, Iwakura K, Watanabe H, Yoshikawa $\mathrm{J}$, et al.; Effect of $\mathrm{ARB} /$ diuretics on diastolic function in patients with hypertension 2 (EDEN2) trial investigators. Effect of diuretic or calcium-channel blocker plus angiotensin-receptor blocker on diastolic function in hypertensive patients. Circ J 2016;80: 426-34.

16. Kario K, Tomitani N, Kanegae H, Ishii H, Uchiyama K, Yamagiwa $\mathrm{K}$, et al. Comparative effects of an angiotensin II receptor blocker (ARB)/diuretic vs. ARB/calcium-channel blocker combination on uncontrolled nocturnal hypertension evaluated by information and communication technology-based nocturnal home blood pressure monitoring - the NOCTURNE study. Circ J 2017;81:948-57.

17. Miura M, Sakata Y, Miyata S, Shiba N, Takahashi J, Nochioka $\mathrm{K}$, et al.; SUPPORT Trial Investigators. Influence of left ventricular ejection fraction on the effects of supplemental use of an- giotensin receptor blocker olmesartan in hypertensive patients with heart failure. Circ J 2016;80:2155-64.

18. Park H, Kim HK, Jeong MH, Cho JY, Lee KH, Sim DS, et al. Clinical impacts of inhibition of renin-angiotensin system in patients with acute ST-segment elevation myocardial infarction who underwent successful late percutaneous coronary intervention. J Cardiol 2017;69:216-21.

19. Lee SE, Kim YJ, Lee HY, Yang HM, Park CG, Kim JJ, et al.; Investigators. Efficacy and tolerability of fimasartan, a new angiotensin receptor blocker, compared with losartan (50/100 mg): a 12-week, phase III, multicenter, prospective, randomized, double-blind, parallel-group, dose escalation clinical trial with an optional 12-week extension phase in adult Korean patients with mild-to-moderate hypertension. Clin Ther 2012;34:552-68, 568.e1-9.

20. Park JB, Sung KC, Kang SM, Cho EJ. Safety and efficacy of fimasartan in patients with arterial hypertension (Safe-KanArb study): an open-label observational study. Am J Cardiovasc Drugs 2013;13:47-56.

21. Correa F, Soto V, Zazueta C. Mitochondrial permeability transition relevance for apoptotic triggering in the post-ischemic heart. Int J Biochem Cell Biol 2007;39:787-98. 